\title{
SIMPLIFIED MULTIPLE CONTINGENCY CALCULATIONS
}

Nathan Keyfitz

Ohio State University, Columbus, Ohio, and Harvard University, Cambridge, Massachusetts, USA

Andrei Rogers

International Institute for Applied Systems Analysis, Laxenburg, Austria

RR-82-30

August 1982

Reprinted from The Journal of Risk and Insurance, volume 49 (1982)

INTERNATIONAL INSTITUTE FOR APPLIED SYSTEMS ANALYSIS Laxenburg, Austria 
Research Reports, which record research conducted at IIASA, are independently reviewed before publication. However, the views and opinions they express are not necessarily those of the Institute or the National Member Organizations that support it.

Reprinted with permission from The Journal of Risk and Insurance 49:59-72, 1982.

Copyright (c) 1982 by American Risk and Insurance Association, Inc.

All rights reserved. No part of this publication may be reproduced or transmitted in any form or by any means, electronic or mechanical, including photocopy, recording, or any information storage or retrieval system, without permission in writing from the copyright holder. 


\section{FOREWORD}

Practically all of formal demography deals with transitions of people from one state at a given moment to another state some time later: for example, single to married, in school to out of school, healthy to sick, in the labor force to out of the labor force, alive to dead. It has now become clear that all such transition patterns may be studied using the mathematical methods developed for the analysis of interregional migration flows within multiregional population systems. In consequence, the mathematical models of multiregional demography adopted by the International Institute for Applied Systems Analysis have been found to be applicable to a wide range of demographic topics. This article illustrates the application of these multistate models to multiple contingency calculations used in actuarial practice.

Related IIASA publications on multistate demography are listed in the back of this report.

ANDREI ROGERS

Chairman

Human Settlements and Services Area 


\title{
Simplified Multiple Contingency Calculations
}

\author{
Nathan Keyfitz and Andrei Rogers
}

\begin{abstract}
Standard life contingency formulas are shown to have matrix analogues. The derivation of these multidimensional forms permits simple solutions to multiple contingency problems, including moves in and out of employment, insurance, marriage, sickness, and retirement. Awkward and inaccurate approximations now commonly used can thus be replaced by matrix formulas readily manipulated by computer.
\end{abstract}

\section{Introduction}

To proceed from life contingencies involving a single decrement of death to the many applications with more than one decrement has customarily involved special treatment for each case. Disability insurance, withdrawals from a life insurance plan, pensions, multiple lives - each has its own section in Jordan [4] and other actuarial texts, with distinct notations and formulas. Ad hoc approximations are introduced for each, some of them awkward. The purpose of this paper is to show how all such multiple contingencies, along with marriage, labor force, and other tables treated by demographers, may formally be handled as special cases of a theorem due to Kolmogorov, with simple matrix formulae, uniform calculations, and consistent approximations.

The simplification begins by expressing all instances as individuals moving from one state to another with specified probability and constituting a Mar-

Nathan Keyfitz is Andelot Professor of Sociology and Demography at Harvard University. A $\mathrm{Ph}$.D. from the University of Chicago, he has also taught at the Universities of Toronto, Chicago, Michigan, Rome, Bombay, Moscow, Vienna, and California, Berkeley. Prior to his college teaching career he worked for 20 years at Statistician and later Senior Research Statistician for the Canadian Dominion Bureau of Statistics. A member of the National Academy of Sciences, he is a Fellow of the Royal Statistical Association. During 1970 he was President of the Population Association of America. He is the author of numerous books, reports, and articles in professional journals.

Andrei Rogers is Chairman of the Human Settlements and Services Area in the International Institute for Applied Systems Analysis located in Laxenburg, Austria. A Ph.D. in City and Regional Planning, he was formerly an Associate Professor of City and Regional Planning at the University of California, Berkeley and Professor of Civil Engineering and Urban Affairs at Northwestern University. He is the author of Introduction to Multiregional Mathematical Demography and three other books.

The authors are more than grateful to Peer Just for numerous calculations, only a few of which are included in this paper, and for his lively comments on our text and formulas. Jan Hoem read the text and made valuable suggestions. Many suggestions of the anonymous referees of the Journal have been included, with resulting improvement in precision and clarity. 
kov process. Thus a person moves in and out of employment, in and out of insurance, of marriage, of sickness, of retirement. As long as the probability of moving is known and depends only on the state in which one is at the time, the methodology can answer questions on such matters as the annual premium for disability insurance (the premium to be paid only while the person is working) and the premium for a pension to a widow if her husband predeceases her (that premium to be paid while the husband is working). These and many other premiums are readily calculable, along with the reserve at each duration of the policy. The methodology is by no means restricted to moves in one direction only; a person can be employed this year, retired next year, and come back into the labor force again the following year. Thus the methodology handles not only decrement tables, but also the more general increment-decrement tables [10].

The formulas that turn up will be simple matrix analogues of those familiar in ordinary life contingencies. As such they not only are easily remembered, in contrast to much of the usual theory of multiple decrement, but also are readily computed. Approximations to handle data given in one-year or fiveyear intervals are straightforward. All these advantages are made accessible by the matrix differential equation due to Kolmogorov that generalizes the familiar

$$
\frac{d I(x)}{d x}=-\mu(x) I(x)
$$

whose solution is

$$
I(x)=\exp \left(-\int_{0}^{x} \mu(a) d a\right)
$$

where $\mu(x)$ is the force of mortality and $l(x)$ the probability of surviving to age $x$.

The general analysis deals not only with the movement from life to death represented by the scalar rate $\mu(x)$ but with the matrix $\mu(x)$, standing for the instantaneous rates of movement between any pair of states that are to be included in the model - between life and death, between two regions of a country, between work and unemployment, between being married and being divorced, between work and disability. Once the basic rates (strictly forces or intensities) of movement for the $\mu_{\sim}^{\mu}(x)$ matrix are known, all else can be found: for example, the chance that a man of 30 who is married will be alive 10 years later and divorced or the chance that a blue-collar person of 25 will be alive and doing white-collar work 30 years later.

The number of states in the Markov chain would be very large if there is one state for every possible cross-classification. The fact that a certain minimum number of cases is required for each cell will in practice place a limit on the number of cells that can be used.

The $\mu(x)$ matrix is to be derived from actual data to a suitable approximation. The off-diagonal elements of $\underset{\sim}{\mu}(x)$ are each the corresponding observed rate of movement in a small time interval with sign reversed. Thus $-\mu_{i j}(x) d x$ 
is minus the intensity of transition with which a person in state $j$ transfers to state $i$ during the short period of time and age $d x$. Each diagonal element of $\underset{\sim}{\mu}(x)$ contains the rate $\mu_{\delta i}$ of dying, with positive sign, along with the total of the off-diagonal elements of the column, $\sum_{i \neq j^{\prime} \mu_{i j}}$, also with positive sign. The reason for this is that the column total has to be conservative - that is, to add to zero with respect to movements of living people among units - since the individual has to go somewhere, or remain in the same state, in each (finite or infinitesimal) transition interval. In short, quantities from the $j$ th state added into the $i$ th state must be subtracted from the $j$ th, so any increment to $\mu_{i j}, i \neq j$, has to be subtracted from $\mu_{j j}$. The net total of each column is the death rate.

Alternatively, one could create an artificial state called "death" and put $\mu_{\delta i}$ in that row, so that the diagonal would represent the intensity of nonmovement of living persons. This is a (multiple) birth-death process type of matrix formulation, or alternatively a Markov process with an absorbing state [5, Chapters 4 and 7].

Table 1 indicates the notation, giving some detail of the matrix $\underset{\sim}{\mu}(x)$. The right-hand subscript is state of origin, the left-hand subscript state of destination. Thus $\mu_{23}(x)$ is the movement from state 3 to state 2 for persons aged $x$, i.e., indexes are read right to left. All other matrices of this article use the same subscripting, essentially that of Rogers [9]. The matrix $\underset{\sim}{M}(x)$ will be the observations that correspond to $\underset{\sim}{\mu}(x)$. The distinction in the algebra is that $\underset{\sim}{\mu}(x)$ is continuous and $\underset{\sim}{\mathrm{M}}(x)=\underset{n \sim X}{\mathrm{M}}$ is in finite intervals of length $n$, where typically $n=5$ years. One could also think of $\underset{\sim}{\mu}(x)$ as a parameter, and ${ }_{5 \sim x}^{\mathbb{M}}$ as a random variable obtained from a sample, but this aspect is not developed in the present paper.

Table 1

Matrix $\underset{\sim}{\mu}(x)$ of Moves

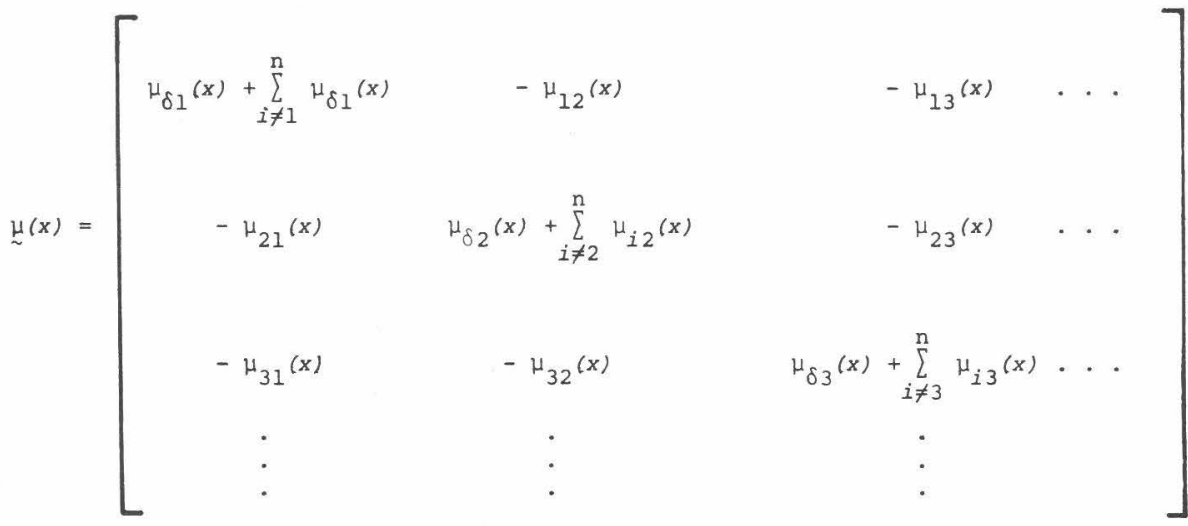


Identical with Equation (1), except that the elements are now matrices and vectors, is the basic

$$
d[\underset{\sim}{I}(x)] / d x=-\underset{\sim}{\mu}(x)[\underset{\sim}{I}(x)]
$$

which is due originally to Kolmogorov $[6,13]$. In our application $[\underset{\sim}{[}(x)]$ is a vertical vector in which the $i$ th element is the number of the population surviving and in the $i$ th category at age $x$. In general, where people are going in and out of the several categories, the ratio of an element of $[l(x)]$ from one $x$ to a preceding $x$ does not represent a probability, yet probabilities are required. Now suppose that in the small interval of time and age $d x$ no one will be affected by more than one event. The objective is to pass from $\mu(x)$ and the vector $[\underset{\sim}{I}(x)]$ to a matrix $\underset{\sim}{I}(x)$ whose typical element is $I_{i j}(x)$, the chance that a person born in the $\tilde{j}$ th state will be in the $i$ th state by age $x$.

The theory for doing this is available from standard works on linear differential equations [1;2, Vol. 2, p. 113]. If there are $n$ states, and so the matrix $\mu(x)$ is $n X n$, and if the $n$ latent roots of that matrix are distinct, there will be $n$ linearly independent vectors $[\underset{\sim}{I}(x)]$ that satisfy Equation (3). When this is so, the matrix made by setting those vectors side by side will obviously also satisfy the equation, and it can be shown to be the complete soultion. Call $\underset{\sim}{I}(x)$ the matrix made up of the several $[I(x)]$. The paper will show next how to obtain the elements of $\underset{\sim}{I}(x)$ so as to ensure that the ijth element is the probability that a person born in the $j$ th category finds himself or herself in the $i$ th category by age $x$.

\section{The Multiplicative Property}

One mathematical property of the $\underset{\sim}{1}(x)$ will be important for the demographic application: its multiplicativity. It may be shown (though not here) that if the interval from zero to $y$ is broken into two subintervals at any point, say $x$ $<y$, [2, Vol. 2, p. 127], then

$$
\underset{\sim}{I}(y)=\underset{\sim}{I}(y \mid x) \underset{\sim}{I}(x),
$$

where the $i j$ th element of $\underset{\sim}{\perp}(y \mid x)$ will mean the probability of being in the $i$ th state at age $y$, given that the person was in the jth state at age $x$. When $y$ is one or five years more than $x$ within the interval $(x, y), \mu(x)$ may be approximated by a matrix whose elements are constants within that age interval. This will be the key to the numerical solution of Equation (3).

If in the interval $(x, x+h), \mu_{i j}(x)$ is constant, say $M_{i j}$, for all $i$ and $j$, and $\underset{\sim x}{M}$ is the array of the $M_{i j}$, from property (4)

$$
\underset{\sim}{I}(x+h)=e^{-h M} \underset{\sim}{\sim} \underset{\sim}{I}(x) .
$$

With an arbitrary radix $l(0)$, Equation (5) permits the construction of $l(x)$ step by step at intervals of $h$ all the way to the end of life. If the $I(x)$ curve takes the form of a declining exponential, which is to say, if the decrement is nearly constant within the age interval, no more need be said. 
But for those parts of the table where the decrement is rising, a somewhat better approximation is obtained by multiplying both sides on the left by $e_{\sim X}^{h M_{X} / 2}$, expanding to the term in $h$, and multiplying by $\left.\underset{\sim}{(I}+\underset{\sim X}{h M} / 2\right)^{-1}$ on the left, to obtain

$$
\left.\underset{\sim}{I}(x+h)=\left(\underset{\sim}{I}+h \underset{\sim}{M} X^{M} / 2\right)^{-1} \underset{\sim}{(I}-h \underset{\sim}{M} / 2\right) \underset{\sim}{I}(x) .
$$

Results (5) and (6) are of course the multidimensional analogues of equations familiar in the ordinary life table. The authors have tested singledecrement data and found (6) to be decidedly superior to (5); intuitively it seems better in general because it is centered on the mid-point of the interval $(x, x+h)$ rather than on the beginning of the interval. The approximation (6) is close enough for many kinds of data with intervals of one year or even five years. It can be improved by graduating the original data down to tenths of a year or smaller. This was essentially what Oechsli $[7,8]$ did, using spline functions.

\section{Probabilities Over Long Intervals}

The next obvious question to ask is: what is the probability that a person in the $j$ th state at age $x$ will find himself or herself in the $i$ th state at age $y$, where the difference $y-x$ need not be small? Without matrix methods the problem is difficult; it has to take account of not only movement out of the $j$ th state but also movement into the $i$ th state of persons not in the $j$ th state at age $x$. The machinery of the matrix approach handles this automatically. The multiplicative property indicates that if $I(y \mid x)$ is the desired set of probabilities, $\underset{\sim}{I}(y \mid x) \underset{\sim}{I}(x)=\underset{\sim}{I}(y)$, so multiplying on the right by ${\underset{\sim}{\sim}}^{-1}(x)$ we have

$$
\underset{\sim}{I}(y \mid x)=I(y) \underset{\sim}{\stackrel{1}{-1}(x),}
$$

where the probability of going from the $j$ th state at age $x$ to the $i$ th state at age $y$ $>x$ is the $j$ th element of the $i$ th row of $\underset{\sim}{I}(y \mid x)$.

\section{Expected Time in the Several States}

Another objective of the paper is to know the expected time lived between age $x$ and $x+h$ in the several states, where in the first instance $h$ is small. In the usual way, call $h_{\sim \sim}^{L}$ the integral of $I(x)$ on the interval $(x, x+h)$ and let discrete values of $\underset{\sim}{I}(x)$ be $\underset{\sim}{I}$. A straight-line approximation gives, as time of residence in the $i$ th state for those initially in the $j$ th state, the $i j$ th element of the matrix $\underset{h \sim x}{L}=(h / 2)\left({\underset{\sim x}{1}}^{L}+{\underset{\sim x}{1}+h}^{1}\right)$, and a cubic gives

$$
\underset{h \sim X}{L}=(13 h / 24)(\underset{\sim X}{L}+\underset{\sim X+h}{1})-(h / 24)\left({\underset{\sim}{x}-h}_{\sim}^{1}+\underset{\sim X}{1}+2 h\right)
$$

Adding $h_{\sim X}^{\mathrm{L}}$ estimates person-years over any interval of age large or small. Cumulating ${ }_{h \sim X}^{L}$ back from the end of the table gives the expected years in the $i$ th state from age $x$ to the end of life measured prospectively from birth in the $j$ th state:

$$
\underset{\sim}{T}(x)=\sum_{X}^{W-h} \underset{\sim}{L}(a)=\int_{X}^{W} \underset{\sim}{I}(a) d a
$$


For an individual just born in the $j$ th state, the probability of being in the $i$ th state by age $x$ is the $i j$ th element of $I(x)$. And if the $k$ th element of $\stackrel{\circ}{e}(x)$ is the expected number of years beyond age $x$ in the $i$ th state for those who survive to the $k$ th state by age $x$,

$$
\underset{\sim}{T}(x)=\underset{\sim}{\stackrel{O}{e}}(x) \underset{\sim}{I}(x)
$$

which gives for the $j$ th state at birth the number of years for which the $i$ th state will be occupied after age $x$.

Consider, for example, those in the second state at birth and find their expectation beyond age $x$ in the first state. The second column of $I(x)$ gives the chance that the person born in the second state is in the first, the second, and so forth, state at age $x$. if residing in the first state at age $x$, he or she has an expected $\stackrel{\circ}{e}_{1 I}(x)$ in the first; and so on. In short the total expectation in the first state, given that the person was born in the second, is prospectively from age zero

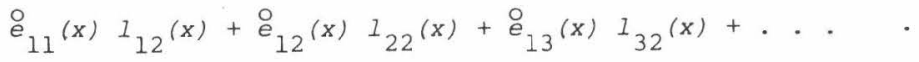

For the whole collection of states,

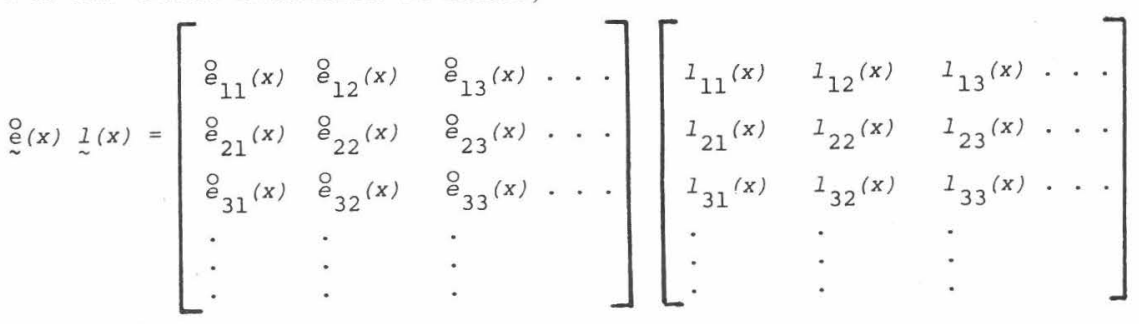

Note that here as in other expressions indexes are read right to left in order to use column vectors and the conventional subscripting of matrix elements.

Premultiplying Equation (9) by ${\underset{\sim}{f}}^{-1}(x)$ on the right, the expectation in the $i$ th state for a person in the $j$ th state at age $x$ is the $i j$ th element of

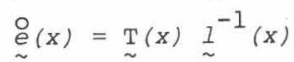

The recursive formulas familiar in the scalar life table are also available in matrix terms. Thus one way of building up the expectation of life is to start at the end of life and work backwards using

$$
\underset{\sim}{\stackrel{O}{e}}(x)=\underset{\sim}{L}(x){\underset{\sim}{I}}^{-1}(x)+\underset{\sim}{\stackrel{\bigcirc}{e}}(x+1) \underset{\sim}{I}(x+1){\underset{\sim}{I}}^{-1}(x)
$$

\section{Marital Status}

Schoen and Nelson [11], Krishnamoorthy [6], and Willekens et al. [14] have presented multidimensional increment-decrement life tables for women by marital status, recognizing the states of never married, married, widowed, and divorced. Working with data for the United States in 1970, Krishnamoorthy [6] finds, for example, that the chance of a child just born being in the never married status at age 50 is 0.034 ; of being married 0.727 ; of being widowed (and not remarried) 0.067; and of being divorced (and not 
remarried) 0.090 . At birth, expected number of years in the never married state is 22.56; married (including remarriages) 32.27; widowed 10.22; divorced 4.71 .

Repeating Krishnamoorthy's calculations with data on Belgian women in 1970-71, Willekens et al. [14] find some differences in the corresponding probabilities and durations. The probability that a baby girl just born is in the never married state by age 50 is 0.034 ; married 0.827 ; widowed 0.043 ; and divorced 0.030 . Expected number of years, at birth, in the never married state is 23.27; married 40.83; widowed 8.81; divorced 1.36. An important finding is that Belgian women, with a divorce rate one fourth that of the United States, exhibit similar proportions of remarriages among divorcees and a much lower one among widows (14 percent versus two percent). Patterns of first marriage are very similar in the two countries, with about 94 percent of the girls born eventually marrying after spending roughly 0.31 of their lifetime in the single (never married) state.

\section{Labor Force Status}

A standard application of the life table concept is to tables of working life [15]. Recently, Hoem and Fong [3], Willekens [13], and Schoen and Woodrow [13] have demonstrated the superiority of the multi-dimensional approach. Using Danish data for 1972-74 Hoem and Fong [3] find, for example, that the chance of a baby boy just born being employed at age 20 is 0.909 , and that the average number of years he can expect to be working during his entire lifetime is $40.98 .^{1}$ Using the same data Willekens [13] calculates that a male unemployed at age 20 can expect to experience about 10.11 years of unemployment, whereas an employed male of the same age can expect the somewhat lower total of 8.04 years.

Repeating Willekens' calculations for females shows significant differences. The average number of future years spent in the employed state by a working 20-year old woman is 29.82 , about 11 years less than the corresponding male figure. The difference at birth is one year less and contrasts markedly with the 15 year difference in the United States reported by Schoen and Woodrow [12], who, comparing the conventional and the multidimensional approaches, conclude that although both give rather similar results for the proportion of total lifetime spent in the labor force, the former conceals a significant amount of movement into and out of the employed state:

While the conventional table shows just under one entry to the labor force per male born, the increment-decrement table shows a lifetime average of $3 \frac{11}{4}$ entries [12, p. 319].

The usual table gratuitously simplifies reality in permitting movement in only one direction - from never having worked into the labor force and from the labor force into permanently retired. Like many other new methods of

\footnotetext{
${ }^{1}$ The data in Hoem and Fong [3] refer to active and inactive statuses, which are not equivalent to being employed and unemployed. Nevertheless, for expositional convenience the two sets of terms will be used interchangeably.
} 
analysis and calculation, this one presses to the limit of available data. Having it available often makes the analysis data-limited rather than theory-limited.

Based on these mathematical and demographic preliminaries, financial calculations can be made as a direct application.

\section{Annuities}

A life annuity of one unit per annum, payable continuously for the rest of life to a person now ages $x$, has the present value

$$
a(x)=\frac{\int_{0}^{w-x} e^{-\delta t} 1(x+t) d t}{l(x)}
$$

where $\delta$ is the force of interest, i.e., the nominal annual rate of interest compounded continuously, and $l(x+t) / l(x)$ is the probability that a person now aged $x$ will be alive $t$ years later. Thus $a(x)$ is the present value of the annuity payable irrespective of whether the person is working or not working, in good health or bad, living in a certain region or not. It is helpful to write $a(x)$ in the form

$$
a(x)=\frac{N(x)}{D(x)}-\frac{\int_{x}^{w} \mathrm{e}^{-\delta t} I(t) d t}{\mathrm{e}^{-\delta x} I(x)}
$$

since $N(x)$ and $D(x)$ need be calculated only once for each $x$, whereas the integrand of (11) would require calculation for all combinations of $x$ and $t$.

Now suppose that the person is to be paid the annuity only if he or she is alive and in a certain state - say, sick, retired, or unemployed. Assume as usual that the condition can be defined precisely and that it is not affected by the annuity. If, for example, the annuity if large enough that an appreciable number of people will change their residence in order to obtain it, place of residence is not a suitable contingency. If the annuity is payable only when the person is sick, there must be some criterion of the sick condition. The application considered here will be the value of the annuity that the person pays to the company or government in the form of a premium or tax while he or she is working, and the different annuity that the person receives while disabled or retired.

Suppose that a person is now aged $x$ and in the $j$ th state, and that the annuity is payable only while he or she is alive and in the $i$ th state. The expected future lifetime in state $i$ for a person initially in state $j$, is provided by the ratio of two matrices given as (10) above

$$
\underset{\sim}{\stackrel{\varrho}{e}}(x)=\underset{\sim}{T}(x){\underset{\sim}{\sim}}^{-1}(x)
$$

If money carries no interest, this is the answer to the annuity problem.

If money carries interest at rate $\delta$ the matrix $\mathrm{T}(x)$ needs to be modified by multiplying all of the $1_{i j}(x+t)$ of which it is made up by $e^{-\delta t}$. The 
discounted matrix ${ }^{\curlyvee}(x)$, with elements $e^{-\delta x} I_{i j}(x)$, can be designated $\underset{\sim}{\mathrm{D}}(x)$, and the corresponding cumulative integral

$$
\underset{\sim}{N}(x)=\int_{0}^{W-x} e^{-\delta(t+x)} \underset{\sim}{I}(x+t) d t
$$

takes the place of the undiscounted $\mathrm{T}(x)$. Following the usual actuarial form everything has been discounted back to age zero. Then the value of an annuity, payable to or by a person age $x$ initially in state $j$, but only while in state $i$, with money discounted at rate of interest $\delta$, is the $i j$ th element of

$$
\underset{\sim}{a}(x)=\underset{\sim}{N}(x) \underset{\sim}{D^{-1}}(x)
$$

A common arrangement is a life annuity plus a condition that the annuity continues for ten (or some other number) years after the policy is taken out. This means in effect an annuity certain for ten years plus a deferred annuity beyond that. The initial value per payment of unity per annum is

$$
\int_{0}^{10} e^{-\delta a} d a+\underset{\sim}{N}(x+10) \underset{\sim}{D^{-1}}(x)=\frac{1-e^{-10 \delta}}{\delta}+\underset{\sim}{N}(x+10) \underset{\sim}{D^{-1}}(x)
$$

In practice annuities and insurance are covered not by single payments but monthly or annually. Suppose continuous payments (any other periodicity can easily be approximated from this) and that payments are made while the person is in state $i$ and an annuity received while the person is in state $h$. In the obvious applications $i$ may be working and $h$ retired; or else $i$ in good health and $h$ in hospital; or $i$ working and $h$ unemployed. Now a premium of unity from a person in the $j$ th state at age $x$, payable as long as he or she is in the $i$ th state, has an initial discounted value given by the $i j$ th element of the matrix $(x)={ }_{N}(x) \mathrm{D}^{-1}(x)$, so a premium of $\mathrm{P}_{h i}$, say, will have an initial discounted value equal to the $i j$ th element of

$$
\mathrm{P}_{h i} \underset{\sim}{\mathrm{a}}(x)=\mathrm{P}_{h i} \underset{\sim}{\mathrm{N}}(x) \underset{\sim}{D^{-1}}(x)
$$

The benefit is another annuity, say of unity, paid each year during tenure in the $h$ th state. For a person in the $j$ th state this benefit must have a present value of the $h j$ th element of $\underset{\sim}{\mathrm{a}}(x)$, say $\mathrm{a}^{(h j)}(x)$. Then the required premium is obtained by equating payment and benefit, i.e.,

or

$$
P_{h i} a^{(i j)}(x)=a^{(h j)}(x)
$$

$$
P_{h i}=a^{(h j)}(x) / a^{(i j)}(x)
$$

Other forms are straightforward enough to be written out immediately. Thus a single-payment endowment, on the life of a person in the $j$ th state, due at age $x+h$, if the person is then in the $i$ th state, is the $i j$ th element of the matrix

$$
\underset{h \sim X}{\mathbb{E}}=\underset{\sim X+h}{\mathrm{D}} \underset{\sim X}{\mathrm{D}^{-1}}
$$

It is often desired to pay only for a certain term at most, without changing the benefit, as in a 20-payment life policy. A 20-payment life policy for disable- 
ment taken out by a person in state $j$, with premiums payable in state $j$ and with benefits receivable in state $i$, would be the $i$ th elements of $\mathrm{N}(x)$ divided by the $j j$ th element of $\mathrm{N}(x)-\mathrm{N}(\mathrm{x}+20)$.

As the simplest of the innumerable examples possible, consider the table of working life calculated by Frans Willekens [13]. His expectations matrix, recognizing only working as state 2 and unemployed as state 1 , is

$$
\underset{\sim}{\stackrel{e}{e}}(17)=\left[\begin{array}{cc}
10.09 & 8.71 \\
41.59 & 42.97
\end{array}\right]
$$

Thus a male employed at age 17 would have an expected 8.71 years unemployed; one unemployed at age 17 would have an expected 10.09 years of unemployment. With a discount throughout at three percent per annum, the same matrix becomes

$$
\underset{\sim}{a}(17)=\left[\begin{array}{rr}
4.54 & 3.22 \\
21.28 & 22.60
\end{array}\right]
$$

so the payment of $\$ 1$ per year while the person is unemployed would have a prospective cost of $\$ 3.22$ for a person initially employed and of $\$ 4.54$ for a person initially unemployed.

If both father and mother have jobs, they may wish to insure their children against the possibility that both fall sick or both die. Matrix expressions can be worked out for such functions of multiple lives.

\section{A Generalized Form of Insurance}

In ordinary insurance practice where living and dead are the only states recognized, the value of an assurance of unity on a life now ages $x$ is

$$
A(x)=\frac{\int_{x}^{W} e^{-\delta t} \mu(t) I(t) d t}{e^{-\delta x} I(x)}=\frac{M(x)}{D(x)}
$$

The general case involves insuring the person against passing from the $i$ th to the $h$ th state, given that he or she is of age $x$ and in the $i$ th state at the time of the insurance. The probability discounted back to time $x$ that the transition will take place at age $y$ is the hith element in

$$
-\bar{\sim}(y) \underset{\sim}{\stackrel{1}{\sim}(y)}{\underset{\sim}{\perp}}^{-1}(x) e^{-\delta(y-x)} d y
$$

where $\bar{\mu}^{(h i)}(y)$ is the probability of transition from $i$ to $h$, given that the person has arrived at state $i$ by age $y .^{2}$ This probability has to be added through all $y>x$. Defining $\underset{\sim}{M}(x)$ as the sum

$$
\underset{\sim}{M}(x)=\int_{x}^{W} \underset{\sim}{\mu}(y) \underset{\sim}{I}(y) e^{-\delta y} d y
$$

\footnotetext{
${ }^{2}$ The numerical approximation of the matrix $\bar{\mu}(y)$ that has been used is $\underset{\sim}{R}(y) \underset{\sim}{L}(y) \underset{\sim}{I}(y)^{-1}$ where is a diagonal matrix with interstate transition rates or death rates in the diagonal.
} 
there being no danger of confusion with the quite different $\underset{\sim}{M}(x)$ matrix containing average rates in a small finite interval, the single payment is

$$
\underset{\sim}{A}(x)=\underset{\sim}{M}(x) \underset{\sim}{D^{-1}}(x)
$$

The insurance of unity taken out by a person in the $i$ th state aged $x$, against arriving into the $h$ th state, is worth the $h i$ th element of $A(x)$. To allow for insurance against the $h$ th or the $k$ th state two such expressions would be added.

If the insurance is to be paid for by an annuity, to find the premium that required element of $\underset{\sim}{A}(x)$ must be divided by an element of $\underset{\sim}{a}(x)$. Suppose the person aged $x$ and now in the $i$ th state, and insurance against passage from the $i$ th to the $h$ th state is to be covered by equal payments while in the $k$ th state. Then the annual premium is

$$
A^{(h i)}(x) / a^{(k i)}(x)
$$

A combined insurance and endowment provides for the payment of a sum to the beneficiary if the person dies within 10 years, and to the person if he or she survives to the end of the 10-year period. The present value of such a policy taken out in state $j$, and insured against falling into state $i$, is the $i j$ th element of

$$
[\underset{\sim}{M}(x)-\underset{\sim}{M}(x+10)+\underset{\sim}{D}(x+10)]{\underset{\sim}{D}}^{-1}(x)
$$

In another type of policy the insured gets his or her premiums back without interest if he or she survives and keeps out of state $i$ for the 10 years. The premium is $\mathrm{P}$ in the following equation:

$P[\underset{\sim}{N}(x)-\underset{\sim}{N}(x+10)] \sim_{\sim}^{D}(x)=[\underset{\sim}{M}(x)-\underset{\sim}{M}(x+10)+10 P \underset{\sim}{D}(x+10)] \sim_{\sim}^{D}(x)$ except that this equation is not to be interpreted as a matrix equation, but only as the $j j$ th element on the left for $\mathrm{D}(x+10) \mathrm{D}^{-1}(x)$, and as the $i j$ th element for the M's. Thus $n(j j)(x)$ woũld be written for the jjth element of $[\mathrm{N}(x)-\tilde{N}(x+10)-10 \mathrm{D}(x+10)] \mathrm{D}^{-1}(x)$, and $m^{(i j)}(x)$ for the $i j$ th element $\tilde{\text { of }}[\underset{\sim}{M}(x) \stackrel{\sim}{\sim} \underset{\sim}{M}(x+10)] \tilde{\sim}^{-1}(x)$. The premium is

$$
P=m^{(i j)}(x) / n^{(j j)}(x)
$$

\section{Reserves}

The contingent liability, say when the person has moved along from age $x$ to age $y$, is available for all such policies. For a single premium annuity taken out at age $x$ and state $j$, and payable while the person is in state $i$, first suppose it is known that the person has moved to the $k$ th state. The reserve needed will be the $i k$ th element of

$$
\underset{\sim}{\mathrm{V}}(y)=\underset{\sim}{\mathrm{a}}(y)=\underset{\sim}{\mathrm{N}}(y) \underset{\sim}{\mathbb{D}^{-1}}(y) .
$$

If one does not know the present state of the individual, the reserve might take account of the probability of the person being in the $k$ th state at age $y$, given that he or she was in the $j$ th state at age $x$, i.e., the $k j$ th element of

$$
\underset{\sim}{1} *(y \mid x)=\underset{\sim}{1}{ }^{*}(y) \underset{\sim}{1} *^{-1}(x)
$$


where the asterisk * means that the 1 matrix has been recalculated omitting the contingency of death - the person is assumed to be alive. The reserve is now

$$
\left.\underset{\sim}{V}(y \mid x)=\underset{\sim}{\mathbb{N}}(y) \underset{\sim}{D^{-1}}(y) \quad \underset{\sim}{[1} *(y \mid x)\right]
$$

In practice it is not of great interest to weight according to the proportions ${ }_{1}{ }^{(k i)}(y \mid x)$ that would be in the several states $k$, given that they were in a certain distribution of state $j$ when they took out the policy. Since those who dropped their policies prior to the time of valuation can be ignored, it is better to weight the several states $k$ according to the business in hand at the time the reserve $j$ when they took out the policy. Since those who dropped their policies prior to the time of valuation can be ignored, it is better to weight the several states $k$ according to the business in hand at the time the reserve is being calculated.

Annuity calculations (13) or (20) give the value of a policy that pays one unit per year of unemployment. For example, if money carries no interest and if the policy is issued to an employed 20-year old Danish male, then 8.04 units is the value of the policy. If the interest rate is three percent, the value declines to 2.84 units and increasing the rate to six percent lowers the value of the policy to 1.40. The reserve still needed to cover the expected payment to, say, a 30-year old man can be calculated using Equation (21). For the Danish data the figure is 2.54 units, if the prevailing rate of interest is three percent, and 1.04 units if it is six percent.

Leaving this somewhat improbable kind of policy, we go on to one in which the payments are made by an annuity while the person is working and benefits obtained while the person is disabled or unemployed. In general to obtain the reserve that would be needed in respect of a person in state $j$ at age $y>x$ recall that the future payments are an annuity of $\mathrm{P}_{h i}$ with expected value per unit equal to the $i j$ th term in $a(y)$, i.e., $\mathrm{P}_{h i} a^{(i j)}(y)$ if the person is still in state $j$. The future benefits are an annuity of unity while a person now in state $j$ is in state $h$, i.e., ${ }^{(h j)}(y)$. Hence the reserve must be

$$
V(y \mid x)=a^{(h j)}(y)-P_{h i} a^{(i j)}(y)
$$

\section{Approximations}

If precise information on transitions is available for single years of age, and in the right form, no question of approximations need arise. Thus when the probability that a man of exact age 31 who is now working will be unemployed one year later is known, along with similar information for all other possible transitions, we need go no futher in order to construct the $I$-matrix. For such ratios give directly what might be called a $\underset{\sim}{\mathrm{p}}$-matrix covering each year separately, that is, $\underset{\sim}{p}(x)=\underset{\sim}{I}(y \mid x)=\underset{\sim}{I}(y){\underset{\sim}{I}}^{-1}(x) \quad$ where $y=x+1$. The cumulative product of the $p$-matrices starting at the beginning of life or any other point gives the probabilities of transitions over long periods of time.

Rarely is information available in such convenient form. At best information might be available concerning exposed population and deaths or other 
transitions over a period of a year. The ratio of cases of transition to exposure was called earlier the $M$ matrix. To go from this matrix to the 1 -matrix has also been discussed abõve, culminating in (6). By integrating the $\frac{1}{\sim}$-matrix, the stationary population, or L -matrix, can be calculated, and from that one can go on to expectations of time spent in the various states. By extension of the recursive formula for $\stackrel{\varrho}{\stackrel{(}{e}(x)}$ the integral for financial calculations in $\delta$-year intervals can be estimated from the $\mathrm{L}$-matrix as follows:

$$
\underset{\sim}{a}(x)=\mathrm{e}^{-\delta / 2} \underset{\sim}{\mathrm{L}}(x) \underset{\sim}{\underset{\sim}{\sim}(x)^{-1}}+\underset{\sim}{\mathrm{e}^{-\delta}} \underset{\sim}{\mathrm{a}}(x+1) \underset{\sim}{\mathrm{p}}(x)
$$

but this seems to add an unnecessary approximation to the process. What should be done instead is to multiply the $\frac{1}{\sim}$-matrix at each age by $\mathrm{e}^{-\delta x}$, where $\delta$ is the rate of interest, and then carry out the integration for $\underset{\sim}{\mathrm{N}}(x)$ just as was done to find expected times $\mathrm{T}(x)$. When a $(x)$ is calculated for a man aged 40 not in the labor force using $(2 \tilde{2})$, the present value of a life insurance of unity at three percent is found to be 0.13295 . The alternative more precise calculation is 0.13302 or about one part in 2000 higher.

\section{Conclusion}

To rewrite standard actuarial formulas in matrix terms is an extensive and potentially useful task. This paper points the way and does a part of the work.

\section{REFERENCES}

1. Coddington, E.A., and N. Levinson, Theory of Ordinary Differential Equations (New York: McGraw Hill, 1955).

2. Gantmacher, F.R., The Theory of Matrices (New York: Chelsea, 1959).

3. Hoem, J., and M. Fong, A Markov Chain Model of Working Life Tables: A New Method for the Construction of Tables of Working Life. Working Paper 2 (Copenhagen: Laboratory of Actuarial Mathematics, University of Copenhagen, 1976).

4. Jordan, C.W., Life Contingencies, 2nd Edition (Chicago: Society of Actuaries, 1975).

5. Karlin, S., and A. Taylor, A First Course in Stochastic Processes (New York: Academic Press, 1975).

6. Krishnamoorthy, S., "The Demography of the Life Cycle," unpublished doctoral dissertation (Cambridge, MA: Harvard University, School of Public Health, 1978).

7. Oechsli, F., "The Parity and Nuptiality Problem in Demography," unpublished doctoral dissertation (Berkeley: University of California, 1971).

8. Oechsli, F., “A Population Model Based on a Life Table That Includes Marriage and Parity,', Theoretical Population Biology, 7 (1975), pp. 229-245.

9. Rogers, A., Introduction to Multiregional Mathematical Demography (New York: John Wiley \& Sons, 1975). 
10. Schoen, R., and K.C. Land, A General Algorithm for Estimating a Markov-Generated Increment-Decrement Life Table with Applications to Marital Status Patterns, Working Papers in Applied Social Statistics WP-77-15 (Urbana: University of Illinois, 1978). Also published in the Journal of the American Statistical Association, 74 (1979), pp. 761-776.

11. Schoen, R., and V.E. Nelson, "Marriage, Divorce, and Mortality: A Life Table Analysis,' Demography, 11 (1974), pp. 267-290.

12. Schoen, R., and K. Woodrow, "Labor Force Status Life Tables for the United States, 1972,'”Demography, 11 (1974), pp. 297-322.

13. Willekens, F., The Demography of Labor Force Participation, Research Memorandum RM-78-17 (Laxenburg, Austria: International Institute of Applied Systems Analysis, 1978). Published in revised form in Environment and Planning $A, 12: 5$ (1980), pp. 563-588.

14. Willekens, F., et al., Multistate Analysis of Marital Status Life Tables. Theory and Application, Working Paper 17 (Voorburg, The Netherlands: Netherlands Interuniversity Demographic Institute, 1980).

15. Wolfbein, S., “The Length of Working Life," Population Studies, 3 (1949), pp. 286-294. 


\section{RELATED IIASA PUBLICATIONS}

Migration Patterns and Population Redistribution

RR-80-7

(Reprinted from Regional Science and Urban Economics)

Andrei Rogers

Essays in Multistate Mathematical Demography

RR-80-10

(Reprinted from a Special Issue of Environment and Planning A)

Andrei Rogers (Editor)

Multidimensionality in Population Analysis

RR-80-33

(Reprinted from Sociological Methodology 1980)

Nathan Keyfitz

Advances in Multiregional Demography

Andrei Rogers (Editor)

RR-81-6 
